\title{
Facial Fat Compartments: A Guide to Filler Placement
}

\author{
Safa E. Sandoval, M.D., ${ }^{1}$ Joshua A. Cox, B.A., ${ }^{2}$ John C. Koshy, M.D., 1 \\ Daniel A. Hatef, M.D., ${ }^{1}$ and Larry H. Hollier, Jr., M.D., F.A.C.S. ${ }^{1}$
}

Advances in anatomic understanding are frequently the basis upon which surgical techniques are advanced and refined. Recent anatomic studies of the superficial tissues of the face have led to an increased understanding of the compartmentalized nature of the subcutaneous fat. This report provides a review of the locations and characteristics of the facial fat compartments and provides examples of how this knowledge can be used clinically, specifically with regard to soft tissue fillers.

KEYWORDS: Fat compartments, facial fat compartments, soft tissue fillers

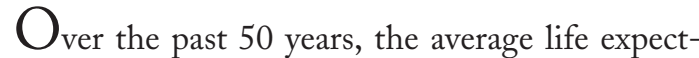
ancy for persons residing in the United States has increased by approximately 10 years. ${ }^{1}$ As a result, today's plastic surgeon is experiencing an increase in the demand for facial rejuvenation procedures among this expanding demographic. Between 1992 and 2008, performance of the rhytidectomy increased by $68 \%{ }^{2}$ Concurrent with these trends has been a strong and steady movement toward minimally invasive procedures. The use of Botox (botulinum toxin, Allergan, Irvine, CA), laser skin resurfacing, and soft-tissue fillers rose by $537 \%, 134 \%$, and $144 \%$ between 2000 and 2008, respectively. ${ }^{2}$ The result of these two trends is that minimally invasive facial rejuvenative procedures are becoming ubiquitous in the field of aesthetic plastic surgery.

Rhytidectomy has undergone a multitude of advancements since its inception in the early 20th century. These changes have paralleled the increased understanding of the anatomy of facial structures. ${ }^{3,4}$ From simple skin excision to subcutaneous, sub-SMAS (superficial muscular aponeurotic system), deep plane, and compo- site dissection, the evolution of the rhytidectomy technique is a testament to the importance of detailed anatomic knowledge in the development and advancement of surgical technique. ${ }^{5-7} \mathrm{~A}$ major advancement that has contributed to the current state of the rhytidectomy is the knowledge that the face does not age as one homogenous object but as many dynamic compartments, which need to be evaluated, augmented, and modified as such. ${ }^{8}$

Obviously, future advancements in facial rejuvenation will be hallmarked by simultaneous progress in the understanding of facial anatomy. Recent landmark studies have determined that not only are the deep facial structures compartmentalized, but the superficial, subcutaneous tissues are as well. The superficial fat compartments are separated by fascial septae, which are nothing but abutments of the fascia that retain these compartments. ${ }^{9-11}$

Just as knowledge of the nature of deep facial structures molded the techniques of rhytidectomy, the new understanding of the subcutaneous tissue of the face should lead to improvements in rejuvenation techniques
${ }^{1}$ Division of Plastic Surgery, Baylor College of Medicine, Texas Children's Hospital, Houston, Texas; ${ }^{2}$ Baylor College of Medicine, Houston, Texas.

Address for correspondence and reprint requests: Larry H. Hollier, Jr., M.D., F.A.C.S., Professor and Program Director, Division of Plastic Surgery, Baylor College of Medicine, Texas Children's Hospital, 6621 Fannin, MC-CC610.00, Houston, TX 77030 (e-mail: 1arryh@bcm.edu).

Contemporary Face-lift Techniques; Guest Editor, Larry H. Hollier, Jr., M.D., F.A.C.S.

Semin Plast Surg 2009;23:283-287. Copyright (C) 2009 by Thieme Medical Publishers, Inc., 333 Seventh Avenue, New York, NY 10001, USA. Tel: +1(212) 584-4662.

DOI 10.1055/s-0029-1242181. ISSN 1535-2188. 
that focus on the skin and superficial tissue, namely the "minimally invasive" injection of fillers and autologous fat. The goal of this report is to present a summary of the recent advancements in understanding of the fat compartments of the face and to highlight the need for further studies that will shed light on how this knowledge can be applied to minimally invasive facial rejuvenation procedures.

\section{METHODS}

\section{Strategy for Literature Review}

MEDLINE, EMBASE, and Cochrane Central Register of Controlled Trials were explored for all articles discussing compartmentalization of the subcutaneous facial structures. The search engines were analyzed from their dates of inception (MEDLINE, 1966; EMBASE, 1974; Cochrane, 2005) through August 2009. The search, utilizing the search terms "Fat" [Mesh] AND "Compartments" [Mesh] AND "Face" [Mesh], produced 37 articles, 11 of which discussed the specific anatomy of facial fat compartments. All articles of interest were reviewed to examine their anatomic findings and discussions. Additional reports were encountered in the discussions of the gathered articles that were also of relevance to the study but were not found during the literature search due to differing nomenclature.

\section{Strategy for Article Selection}

Articles included were those that used cadaver or in vivo dye injections or dissection techniques to explore one or more facial fat component. Articles that did not collect data in these manners were not included. Only articles published in journals written in the English language were included.

\section{RESULTS}

\section{Forehead}

In 2007, Rohrich et al determined, via dye injection in cadaver heads, that there are three fat compartments of the forehead. The central compartment is located in the midline and extends inferiorly to the nasal dorsum and laterally to a border that they refer to as the "central temporal septum." Moss et al describe the location of the middle fat compartments as just lateral to the "central temporal septum," inferior to the orbicularis retaining ligament (ORL), and lateral to the superior temporal septum. ${ }^{13}$ The lateral-temporal fat compartment is located adjacent to the superior temporal septum and is contiguous and confluent with the lateral subcutaneous cheek fat and subcutaneous cervical fat. ${ }^{12}$

\section{Upper and Lower Eyelids (Postseptal, Pre- levator Muscle)}

In 1951, Castanares provided the first documented description of the intraorbital fat compartments: two superiorly and three inferiorly. ${ }^{14}$

In 1977, Barker presented a case series in which he performed in vivo dye injections in patients undergoing upper eyelid blepharoplasty and confirmed that the eyelid fat was compartmentalized into medial and lateral, nonconfluent compartments. ${ }^{15}$

In 1988, Gradinger described a third fat compartment in the upper eyelid, which was noted in patients who presented for secondary blepharoplasty with a prominent lateral bulge. ${ }^{16}$ In 1991, Niechajev and Ljungqvist confirmed the finding of a third upper eyelid compartment in a similar population of patients who presented for revisional blepharoplasty. ${ }^{17}$

More recently, in 1997, Ullmann et al performed cadaveric dissections and grossly identified a third compartment in all of the cadavers dissected. Notably, the third compartment was in close proximity and sometimes attached to the medial fat compartment in its posterior extension. Although the degree of separation of these two compartments varied between cadavers, two distinct compartments were visualized on histologic examination in all of the cadavers. ${ }^{18}$

\section{Subcutaneous Periorbital Area}

In their 2007 cadaveric study, Rohrich et al found three subcutaneous compartments located around the periphery of the eye. The superior compartment is bounded inferiorly by the ORL and extends medially and laterally to the medial and lateral canthi, respectively. The inferior compartment is bounded superiorly by the insertion of the orbital septum into the inferior lid tarsus and inferiorly by the ORL; it extends in an arc from its origin along the orbital rim, where it then inserts into the dermis and is further bounded by the medial and lateral canthi at its medial- and lateral-most aspects, respectively. The lateral compartment is bounded superiorly by the inferior temporal septum and inferiorly by the septum that is referred to as the "superior cheek septum." Notably, the zygomaticus major (ZM) muscle is attached to this fat compartment. ${ }^{12}$

\section{Superficial Cheek}

In their 2007 study, Rohrich et al injected dye into cadaveric heads and determined that there are four superficial fat compartments in the cheek area: nasolabial, medial, middle, and lateral-temporal. The nasolabial fat compartment is bounded medially by the nasolabial crease and is reported to be bounded superiorly by the ORL. The ZM muscle also adheres to this compartment. Notably, the volume of this compartment 


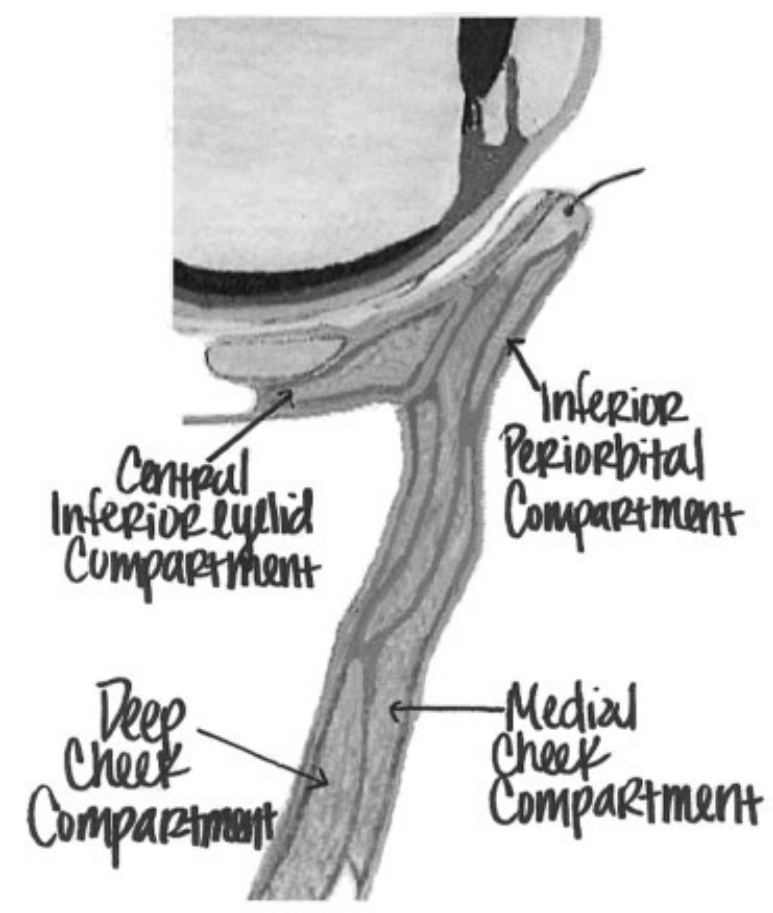

Figure 1 A parasagittal view through the orbit and cheek, demonstrating the relationships between the deep inferior eyelid compartment, sub-orbicularis oculi fat (SOOF), superficial inferior periorbital fat, superficial medial cheek fat, and deep cheek fat. The superficial periorbital compartment extends inferiorly to the orbicularis retaining ligament, which is not labeled. The deep and medial cheek compartments both extend superiorly to the malar septum, which is not labeled.

was reported to be consistent across cadavers. The medial fat compartment is located just lateral to the nasolabial fat (described earlier) and is bounded superiorly by the ORL and laterally by the subcutaneous periorbital compartment and inferiorly by the jowl fat. The middle is located just lateral to the medial compartment, anterior and superficial to the parotid gland, and extends laterally to what can be referred to as the "lateral cheek septum" (Fig. 1). Notably, the ZM is adherent to this compartment as well, in the form of the well-known zygomatic ligament. The lateral-temporal is the most lateral cheek compartment, is located immediately superficial to parotid, and, as previously described, connects the lateral forehead fat to the subcutaneous cervical fat. $^{12}$

\section{Deep Cheek}

In 2008, Rohrich et al discovered the deep medial cheek fat compartment that is located just medial to the buccal fat pad and ZM muscle. The compartment is bounded medially by the pyriform ligament of the nasal base, superiorly by the ORL, and lies just deep to the orbicularis oculi muscle. The compartment is distinct from the sub-orbicular's oculi fat SOOF and abuts the superior sub-orbicularis oris fat inferiorly. A portion of the compartment surrounds the levator anguli oris muscle. The compartment is located just deep to both the medial and middle superficial cheek compartments. The compartment extends posteriorly to the maxillary periosteum but leaves a potential space that is referred to as Ristow's space. ${ }^{19}$

Further dissections found that the deep medial cheek fat compartment can also be seen as two distinct compartments: one that abuts the pyriform membrane (medially), and the other that surrounds the levator anguli and is adjacent to the buccal fat (laterally). ${ }^{19}$

\section{Perioral Area}

In two individual case series of cadaver dissections Rohrich et al identified a deep fat compartment of both the upper and lower lips that is located deep to the orbicularis oris muscle. The orbicularis oris muscle inserts into the dermis at the wet-dry border, and the deep perioral fat compartments are located immediately posterior to this insertion. ${ }^{20}$

\section{Jawline}

In 2008, Reece et al found that the jowl is formed by the deflation or descent of two fat compartments that line the jaw. These fat compartments were labeled the superior and inferior jowl fat compartments. These are distinctly highlighted by methylene blue dye injection, and they are noted to be bounded medially by the labiomandibular crease, where the lip depressors such as the depressor anguli oris insert. Interestingly, these fat compartments share an inferior border at the mandible where membranous fusion of the platysma occurs. ${ }^{21}$ This is now known as the mandibular septum (previously recognized as the mandibular retaining ligament by Furnas).

\section{Chin}

In 2009, Rohrich et al found a deep chin fat compartment located deep to the mentalis muscle and spanning the border of the lower lip. Notably, this compartment is located on either side of the midline, over which it was not confluent. This compartment is distinct from the suborbicularis oris fat compartment that lies superiorly. ${ }^{20}$

\section{DISCUSSION}

As people age, many are left with the feeling that their physical appearance is no longer an accurate representation of their mental state, leaving them with the desire for facial rejuvenation, or "matching their outsides with their insides." The face, like other parts of the body, ages in a unique but relatively consistent manner, creating a 
distinct and easily recognizable "aged" appearance. ${ }^{4}$ The typical aged face probably represents a combination of changes on many levels, including changes in bony structures, fat volume, muscle and ligament strength, skin properties, and vascularity. Many verbs have been used to describe the underlying mechanical changes with aging, including deflation, attenuation, "doubling over," radial expansion, and rotation. ${ }^{21-24}$

In a landmark article in 1965, Gonzalez-Ulloa and Flores describe the process of aging as resulting from the gradual absorption of fat, decreased thickness and elasticity of skin, decreased adherence between the skin and subcutaneous tissue, sagging of the soft tissues, weakening of the orbital muscle and septae, and the progressive decrease in the volume of the craniofacial skeleton. ${ }^{25} \mathrm{~A}$ recent study by Guyuron et al highlights the importance of environmental factors in the aging process by analyzing identical twins that led different lifestyles. Smoking and sun exposure were significantly associated with an older appearance, and the use of hormone replacement therapy was associated with a younger appearance. Interestingly, a higher body mass index (BMI) correlated with an older appearance up to age 40 , after which it was associated with a younger appearance. This fact highlights the importance of adipose tissue in the aged appearance. ${ }^{26}$

Knowledge of the subcutaneous, superficial structure of the face has not yet been utilized in an extensive way in the field of minimally invasive facial rejuvenation. Lasers, chemical peels, and injectable fillers are typically used either diffusely over the face or over specific regions of concern that are visible to the naked eye, such as rhytides and areas of hyperpigmentation.

A specific example of how knowledge of the superficial structures of the face can augment current minimally invasive procedures is suggested by Rohrich et al in their study of perioral fat compartments. ${ }^{20}$ Rohrich et al suggest that the typical "overfilled" and undesirable appearance of the lips after the injection of fillers is due to a lack of understanding of the compartmentalized nature of the lips. Currently, the established area for injection of fillers in the lip is the vermillioncutaneous border in a vertical direction, which places the filler into the superficial compartment of the lip, which is anterior to the orbicularis oris muscle. They suggest, based on the results of their cadaveric studies, that if fillers are injected in a posterior direction, the filler will enter the deeper lip compartment, which is posterior to the orbicularis oris muscle, and will provide a less "overfilled" appearance, cause the lip to evert slightly, and cause increased show of the wet-dry junction, which are all signs of a youthful lip (Fig. 2).

Another example of a potential change in minimally invasive procedures was demonstrated in the study of the deep medial fat compartment of the cheek by Rohrich et al. ${ }^{19}$ When saline was injected into the deep

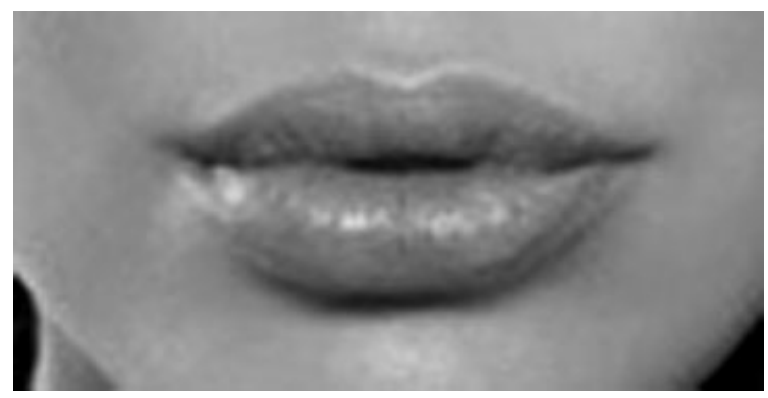

Figure 2 A photograph of a youthful lip, demonstrating fullness, slight eversion of the lower lip, visibility of the wetdry junction of the lower lip, and preserved vertical rhytides.

medial fat compartment, as opposed to superficially in the area of the nasolabial fold, numerous changes occurred, including an increase in anterior projection of the midface, flattening of the nasojugal and nasolabial creases, and an overall improved appearance of the malar region. These changes, all brought about by manipulation of one fat compartment, highlight the dynamic nature of the aging face.

A major limitation that is present in all of the studies presented is the lack of longitudinality. Longitudinal studies that quantify the changes in each facial compartment relative to each other over time will shed light on which individual compartments can be targeted in facial rejuvenation. Eventually, as longitudinal studies are undertaken, we will know more regarding the dynamic changes that occur within and between each fat compartment with aging. Until that point, care should be taken to analyze the aging face not only by the overall appearance of the subcutaneous fat but also based on the anatomic map of the subcutaneous fat compartments.

\section{CONCLUSION}

The recent anatomic literature surrounding the concept of fat compartments of the face is reviewed, and a global understanding of their anatomy is given. Just as previous advances in facial anatomy served as the foundation for more aggressive and long-lasting face-lift techniques, the authors posit that this new conceptual understanding of the facial fat will lead to new and improved methods of autologous fat injection. These fat compartments may serve as the "GPS" for the injection of facial fillers. Future studies at our institution will focus on the effect of fillers on the fat compartments and the visual changes created by their augmentation.

\section{REFERENCES}

1. National Center for Health Statistics. Health, United States, 2006 with Chartbook on Trends in the Health of Americans. Hyattsville, MD: National Center for Health Statistics; 2006 
2. American Society of Plastic Surgeons. National clearinghouse of plastic surgery statistics: 2009 report of 2008 statistics. Available at: http://www.plasticsurgery.org/media/press_kits/ procedural_statistics.html. Accessed Aug. 04, 2007

3. Rogers BO. The development of aesthetic plastic surgery: a history. Aesthetic Plast Surg 1976;1:3-24

4. Gonzales-Ulloa M. The history of rhytidectomy. Aesthetic Plast Surg 1980;4:1-45

5. Lemmon ML, Hamra ST. Skoog rhytidectomy: a five-year experience with 577 patients. Plast Reconstr Surg 1980;65: 283-297

6. Hamra ST. The deep-plane rhytidectomy. Plast Reconstr Surg 1990;86:53-61; discussion 62-63

7. Hamra ST. Composite rhytidectomy. Plast Reconstr Surg 1992;90:1-13

8. Donofrio LM. Fat distribution: a morphologic study of the aging face. Dermatol Surg 2000;26:1107-1112

9. Rohrich RJ, Pessa JE. The retaining system of the face: histologic evaluation of the septal boundaries of the subcutaneous fat compartments. Plast Reconstr Surg 2008;121:18041809

10. Schaverien MV, Pessa JE, Rohrich RJ. Vascularized membranes determine the anatomical boundaries of the subcutaneous fat compartments. Plast Reconstr Surg 2009;123: 695-700

11. Hatef DA, Hollier LH Jr. Vascularized membranes determine the anatomical boundaries of the subcutaneous fat compartments. Plast Reconstr Surg 2009;124:315-316

12. Rohrich RJ, Pessa JE. The fat compartments of the face: anatomy and clinical implications for cosmetic surgery. Plast Reconstr Surg 2007;119:2219-2227; discussion 2228-2231

13. Moss CJ, Mendelson BC, Taylor GI. Surgical anatomy of the ligamentous attachments in the temple and periorbital regions. Plast Reconstr Surg 2000;105:1475-1490; discussion 1491-1498

14. Castanares S. Blepharoplasty for herniated intraorbital fat: anatomical basis for a new approach. Plast Reconstr Surg 1951; 8:46-58
15. Barker DE. Dye injection studies of intraorbital fat compartments. Plast Reconstr Surg 1977;59:82-85

16. Gradinger GP. Cosmetic upper blepharoplasty. Clin Plast Surg 1988;15:289-297

17. Niechajev IA, Ljungqvist A. Central (third) fat pad of the upper eyelid. Aesthetic Plast Surg 1991;15:223-228

18. Ullmann Y, Levi Y, Ben-Izhak O, Har-Shai Y, Peled IJ. The surgical anatomy of the fat in the upper eyelid medial compartment. Plast Reconstr Surg 1997;99:658-661

19. Rohrich RJ, Pessa JE, Ristow B. The youthful cheek and the deep medial fat compartment. Plast Reconstr Surg 2008;121: 2107-2112

20. Rohrich RJ, Pessa JE. The anatomy and clinical implications of perioral submuscular fat. Plast Reconstr Surg 2009;124: 266-271

21. Reece EM, Pessa JE, Rohrich RJ. The mandibular septum: anatomical observations of the jowls in aging-implications for facial rejuvenation. Plast Reconstr Surg 2008;121:14141420

22. Pessa JE. An algorithm of facial aging: verification of Lambros's theory by three-dimensional stereolithography, with reference to the pathogenesis of midfacial aging, scleral show, and the lateral suborbital trough deformity. Plast Reconstr Surg 2000;106:479-488

23. Lambros V. Observations on periorbital and midface aging. Plast Reconstr Surg 2007;120:1367-1376; discussion 1377

24. Pessa JE, Zadoo VP, Mutimer KL, et al. Relative maxillary retrusion as a natural consequence of aging: combining skeletal and soft-tissue changes into an integrated model of midfacial aging. Plast Reconstr Surg 1998;102:205-212

25. Gonzalez-Ulloa M, Flores ES. Senility of the face: basic study to understand its causes and effects. Plast Reconstr Surg 1965;36:239-246

26. Guyuron B, Rowe DJ, Weinfeld AB, Eshraghi Y, Fathi A, Iamphongsai S. Factors contributing to the facial aging of identical twins. Plast Reconstr Surg 2009;123:13211331 